\title{
Les rencontres singulières entre les élèves présentant des difficultés d'apprentissage en mathématiques et leurs enseignants
}

\section{Remarkable Meetings Between Students with Learning Difficulties in Mathematics and Their Teachers \\ Los encuentros singulares entre los alumnos que presentan dificultades de aprendizaje en matemáticas y sus maestros}

\section{Gisèle Lemoyne et Geneviève Lessard}

Volume 31, numéro 2, automne 2003

La spécificité de l'enseignement des mathématiques en adaptation scolaire

URI : https://id.erudit.org/iderudit/1079586ar

DOI : https://doi.org/10.7202/1079586ar

\section{Aller au sommaire du numéro}

Éditeur(s)

Association canadienne d'éducation de langue française

ISSN

0849-1089 (imprimé)

1916-8659 (numérique)

Découvrir la revue

Citer cet article

Lemoyne, G. \& Lessard, G. (2003). Les rencontres singulières entre les élèves présentant des difficultés d'apprentissage en mathématiques et leurs

enseignants. Éducation et francophonie, 31(2), 13-44.

https://doi.org/10.7202/1079586ar

\section{Résumé de l'article}

La question des difficultés d'apprentissage en mathématiques d'un nombre important d'élèves et des mesures à mettre en place pour transformer cette situation est un objet sensible dans tout système éducatif. Elle est aussi, depuis plusieurs décennies, au centre des préoccupations d'un grand nombre de chercheurs. Pour rendre compte de la complexité du traitement de cette question, nous avons choisi de répondre aux questions suivantes: Comment le traitement de cette question a-t-il évolué depuis les dernières décennies? Quelles sont les théories et les recherches qui ont marqué cette évolution? Comment l'enseignement des mathématiques en adaptation scolaire a-t-il évolué ou se positionne-t-il en regard de l'enseignement des mathématiques en classes régulières? Quelles sont les avenues prometteuses d'enseignement aux élèves présentant des difficultés d'apprentissage en mathématiques? Quelles sont les difficultés auxquelles sont confrontés les enseignants en adaptation scolaire? Quelles sont les attentes et les pratiques en classes régulières et spéciales des futurs enseignants en adaptation scolaire? Comment évoluent-elles au cours de leur formation? Les réponses que nous proposons prennent appui sur des recherches réalisées en psychologie, en éducation et en didactique des mathématiques, et sur certaines des expériences que nous avons nous-mêmes effectuées.
Tous droits réservés (C) Association canadienne d'éducation de langue française, 2003
Ce document est protégé par la loi sur le droit d'auteur. L'utilisation des services d'Érudit (y compris la reproduction) est assujettie à sa politique d'utilisation que vous pouvez consulter en ligne. 


\section{Les rencontres singulières entre les élèves présentant des difficultés d'apprentissage en mathématiques et leurs enseignants}

Gisèle LEMOYNE

Département de didactique, Université de Montréal, Montréal (Québec), Canada

Geneviève LESSARD

Département de didactique, Université de Montréal, Montréal (Québec), Canada

\section{RÉSUMÉ}

La question des difficultés d'apprentissage en mathématiques d'un nombre important d'élèves et des mesures à mettre en place pour transformer cette situation est un objet sensible dans tout système éducatif. Elle est aussi, depuis plusieurs décennies, au centre des préoccupations d'un grand nombre de chercheurs. Pour rendre compte de la complexité du traitement de cette question, nous avons choisi de répondre aux questions suivantes : Comment le traitement de cette question a-t-il évolué depuis les dernières décennies? Quelles sont les théories et les recherches qui ont marqué cette évolution? Comment l'enseignement des mathématiques en adaptation scolaire a-t-il évolué ou se positionne-t-il en regard de l'enseignement des mathématiques en classes régulières? Quelles sont les avenues prometteuses d'enseignement aux élèves présentant des difficultés d'apprentissage en mathématiques? Quelles sont les difficultés auxquelles sont confrontés les enseignants en adaptation 
scolaire? Quelles sont les attentes et les pratiques en classes régulières et spéciales des futurs enseignants en adaptation scolaire? Comment évoluent-elles au cours de leur formation? Les réponses que nous proposons prennent appui sur des recherches réalisées en psychologie, en éducation et en didactique des mathématiques, et sur certaines des expériences que nous avons nous-mêmes effectuées.

\section{ABSTRACT}

\section{Remarkable Meetings Between Students with Learning Difficulties in Mathematics and Their Teachers}

The question of the learning difficulties many students have with mathematics and the measures needed to change this situation is a sensitive issue for the entire education system. For several decades, it has also been the main focus of many researchers. To raise awareness of the complexity of the treatment of this question, we chose to answer the following questions: How has the handling of this question evolved over recent decades? What theories and research have stood out during this evolution? How has teaching mathematics to students with special needs evolved or positioned itself in relation to teaching mathematics in regular classes? What are some promising avenues for teaching students with learning difficulties in mathematics? What difficulties are teachers confronted with in special education? What are the expectations and practices in the regular and special classes of future special education teachers? How will they evolve over the course of their training? We support the answers we offer with research from the fields of psychology, education and the didactics of mathematics and on some experiments we carried out ourselves.

\section{RESUMEN}

\section{Los encuentros singulares entre los alumnos que presentan dificultades de aprendizaje en matemáticas y sus maestros}

La cuestión de las dificultades de aprendizaje en matemáticas de una gran cantidad de alumnos y las medidas tomadas para transformar esta situación constituyen un aspecto sensible en todo sistema educativo. Dicha cuestión también constituye, desde hace varias décadas, el centro de las preocupaciones de un gran numero de investigadores. Con el fin de describir la complejidad del tratamiento de este problema, hemos decidido ofrecer una respuesta a las cuestiones siguientes : ¿Cómo ha evolucionado el tratamiento de esta cuestión durante los últimos decenios? ¿Cuales han sido las teorías y las investigaciones que han dejado una huella en dicha evolución? ¿Cómo ha evolucionado la enseñanza de las matemáticas en adaptación escolar y cómo se sitúa con respecto a la enseñanza de las matemáticas en las clases regulares? ¿Cuales son las avenidas más prometedoras para enseñar a 
los alumnos que presentan dificultades de aprendizaje en matemáticas? ¿Cuales son las dificultades que confrontan los maestros en adaptación especial? ¿Cuales son las expectativas y las practicas en clases regulares y especiales de los futuros maestros en adaptación especial? ¿Cómo se transforman durante la formación? Las respuestas que proponemos se apoyan en las investigaciones que se han realizado en sicología, en educación y en didáctica de las matemáticas, así como en ciertas experiencias que nosotros mismas hemos efectuado.

\section{Introduction}

Traiter de l'enseignement des mathématiques en adaptation scolaire n'est pas chose facile. Qui dit adaptation scolaire dit à la fois volonté d'adapter l'école aux besoins de clientèles particulières d'élèves et volonté d'adapter ces clientèles d'élèves aux exigences de l'école. Les documents ministériels, les programmes d'études, les projets éducatifs des écoles, les politiques relatives à l'embauche d'orthopédagogues et d'autres professionnels scolaires, les pratiques d'enseignement et d'apprentissage en vigueur dans les écoles, montrent diverses tentatives pour parvenir à cette adaptation duale. Dans ce texte, nous nous intéressons plus spécifiquement à l'enseignement des mathématiques auprès d'élèves présentant des difficultés d'apprentissage.

Les élèves présentant des difficultés d'apprentissage constituent l'effectif le plus important de la population en adaptation scolaire au Québec, depuis près de trois décennies (Matte (1984); Tardif et Presseau (2000). Les fluctuations de cet effectif au fil des ans ne sont pas fortuites. Elles dépendent, entre autres, des objectifs éducatifs mis de l'avant par le ministère de l'Éducation et des sommes allouées aux institutions scolaires pour l'embauche de professionnels. L'augmentation de la population d'élèves présentant des difficultés d'apprentissage a ainsi été plus significative entre 1960 et 1980 qu'entre 1980 et aujourd'hui. Par ailleurs, depuis les dernières années, on assiste à une augmentation sensible de cet effectif dans le secteur de l'adaptation scolaire de l'enseignement secondaire. Ce phénomène n'est pas unique au Québec. Comme le montre Chevallard (1988), l'attribution d'échec scolaire par les responsables de l'éducation en France est également conjoncturelle. Ainsi, depuis les deux dernières décennies, la demande pour des citoyens qualifiés, aptes à occuper des postes dans divers secteurs industriels et technologiques, a fait en sorte que les préoccupations concernant l'échec scolaire (ou les difficultés d'apprentissage) se sont déplacées de l'enseignement élémentaire à l'enseignement secondaire.

Depuis plusieurs décennies, l'identification des difficultés d'apprentissage préoccupe nombre de chercheurs en éducation et en sciences cognitives. Et pour cause! Comment expliquer que, dans des conditions scolaires équivalentes, certains élèves obtiennent des résultats académiques satisfaisants, tandis que d'autres ne 
parviennent pas à réaliser les apprentissages attendus? C'est pour répondre à cette question qu'on a cherché à caractériser le fonctionnement des élèves présentant des difficultés et construit des outils théoriques et méthodologiques permettant de poser un diagnostic et de suggérer diverses approches remédiatrices.

Déplorant la prolifération de termes suggérés par les chercheurs pour effectuer un diagnostic des difficultés d'apprentissage, Fry (1968) : voir Farhnam-Diggory (1979, p. 14), traduction libre) propose un système diagnostique, en ajoutant, non sans une certaine pointe d'ironie, ce qui suit :

"Le système qui suit donnera 1000 termes mais si ce n'est pas suffisant vous pouvez utiliser la dyslexie, l'aphasie, la neurophrénie, etc. ». Voici les termes proposés par Fry et la façon de les combiner pour identifier les difficultés d'apprentissage (C.N.S. Système Nerveux Central) : "Choisir un mot de la colonne 1, ajouter un mot dans la colonne 2 et un autre mot de la colonne 3. Si vous n'aimez pas le résultat, essayez à nouveau. La signification sera sensiblement la même."

\begin{tabular}{|c|c|c|}
\hline Qualificatif & Aire d'implication & Problème \\
\hline Minimal & Cerveau & Dysfonctionnement \\
\hline Minimal & Cerveau & Dysfonctionnement \\
\hline Modéré & Cérébral & Dommage \\
\hline Mineur & Neurologique & Désordre \\
\hline Chronique & Arithmétique & Désynchronisation \\
\hline Diffus & C.N.S. & Handicap \\
\hline Spécifique & Langage & Inhabilité \\
\hline Primaire & Lecture & Retard \\
\hline Désorganisé & Perception & Défaut \\
\hline Organique & Impulsion & Pathologie \\
\hline Confus & Conduite & Syndrome \\
\hline
\end{tabular}

À la même époque, Cruickshank (1967) : voir Farhnam-Diggory (1979, p. 17, traduction libre) écrit :

"Si un enfant vit dans l'état du Michigan, les éducateurs le qualifient d'enfant qui présente une inhabilité perceptive. Si ce même enfant est un résidant de la Californie, son éducation peut être prise en charge s'il entre dans la catégorie d'enfant handicapé "éducationnellement ou neurologiquement». En Pensylvanie, ce même enfant sera placé dans une classe pour enfants présentant des problèmes de langage. "

Peut-on affirmer que les propositions précédentes, qui semblent aujourd'hui étonnantes, ne trouvent pas d'équivalents dans les conceptions qui sous-tendent certaines des propositions actuelles? Quelle que soit notre réponse à cette question, nous devons admettre que ces propositions montrent bien comment la question des difficultés d'apprentissage, que ces difficultés soient en français ou en mathématiques, est une question sensible dans tout système éducatif. 
- Comment le traitement de cette question a-t-il évolué depuis les dernières décennies? Quelles sont les théories et les recherches qui ont marqué cette évolution?

- Comment l'enseignement des mathématiques en adaptation scolaire a-t-il évolué ou se positionne-t-il en regard de l'enseignement des mathématiques en classes régulières? Quelles sont les avenues prometteuses d'enseignement aux élèves présentant des difficultés d'apprentissage en mathématiques.

- Quelles sont les difficultés auxquelles sont confrontés les enseignants en adaptation scolaire?

- Quelles sont les attentes et les pratiques en classes régulières et spéciales des futurs enseignants en adaptation scolaire? Comment évoluent-elles au cours de leur formation?

Voilà quelques questions qui animent notre travail.

\section{Les études sur le diagnostic et la rééducation des difficultés des élèves en mathématiques}

Dans notre rétrospective des principales études sur le diagnostic et la rééducation des difficultés des élèves en mathématiques, nous distinguons les études réalisées avant et après 1980. Ce découpage relativement grossier nous apparaît correspondre à des transformations ou changements de paradigmes.

Les études antérieures à 1980 partageaient une motivation et une approche naturelles et non négligeables avec d'autres études en sciences appliquées : chercher à résoudre de réels problèmes dont souffraient des individus. Les résultats peu probants des interventions découlant de ces études et l'entrée, autour des années 80, d'approches pédagogiques inspirées par les théories en sciences cognitives, ont conduit les chercheurs à examiner les difficultés des élèves en relation avec les dispositifs d'enseignement, et à proposer des adaptations de ces dispositifs. Le passage à une vision plus « systémique » des difficultés d'apprentissage est aujourd'hui réalisé. La prise en compte des théories en didactique des mathématiques (Artigue (1990), (1994); Bergeron et Herscovics (1989); Brousseau (1998); Brun (1994); Centeno (1995); Chevallard (1991), (1996); Conne (1996); Mercier (1992), (1995); PerrinGlorian (1993); Rouchier (1991); Vergnaud (1991), (1996)) a permis de mieux comprendre le fonctionnement du système didactique et de ses sous-systèmes, de mieux lier difficultés d'apprentissage et d'enseignement. Diverses ingénieries didactiques ont été construites pour l'enseignement régulier. À notre connaissance, aucune ingénierie pour l'enseignement en classes d'adaptation n'a été réalisée. Ce fait soulève des questions sur la spécificité de l'enseignement des mathématiques en adaptation scolaire, sur la singularité des rencontres élèves-enseignants-mathématiques dans les classes spéciales. 


\section{Première période : antérieure à 1980}

Les études sur les difficultés d'apprentissage sont relativement anciennes. Nous donnons un aperçu de quelques études déterminantes.

\section{Définitions, manifestations, diagnostics des difficultés d'apprentissage ou des troubles de l'apprentissage}

Les difficultés d'apprentissage des personnes ayant subi des traumatismes cérébraux (notamment, durant la seconde guerre mondiale) ont retenu l'attention de plusieurs chercheurs. Les descriptions des manifestations de ces difficultés ne sont pas sans évoquer certaines des descriptions que l'on rencontre encore aujourd'hui. Rappelons certaines des descriptions proposées par Strauss et Werner (1942) : voir Farhnam-Diggory (1979) :

a. Réponses amplifiées aux stimuli : tout bruit, tout mouvement ou tout objet retiennent immédiatement l'attention.

b. Fixation pathologique: persévérance dans des actions, malgré les résultats négatifs de ces actions; on peut associer ce défaut au défaut précédent: des stimuli continuent de capter l'attention.

c. Absence d'inhibition : activité motrice excessive, hyperactivité.

d. Dissociation des informations : absence d'intégration des informations ou incapacité de relier les informations en un tout cohérent.

Durant la même période, Luria (1966) : voir Farhnam-Diggory (1979, pp. 84-91) s'est aussi intéressé aux difficultés en arithmétique d'adultes ayant subi des dommages cérébraux. Ses travaux sont précurseurs des travaux prenant en compte le système de traitement de l'information. Les difficultés en arithmétique sont ainsi associées à des défaillances multiples :

a. Défaut de logique spatiale : par exemple, un adulte écrit le nombre 1029 ainsi : 120 ou 100029.

b. Défaut dans la planification : par exemple, le problème «Un garçon est âgé de huit ans. Son père a trente ans de plus que lui et sa mère est dix ans plus jeune que le père. Quel est l'âge de chacun. " est traité de la façon suivante : chaque partie doit avoir 30, puis 10 et enfin 8, ce qui fait 48 ans et si on divise par trois...

c. Persévérance dans l'utilisation de procédures qui ne sont plus appropriées. Par exemple, le problème "Sur deux rayons d'une étagère, il y a 18 livres. Mais ces livres ne sont pas répartis également. Sur un des rayons, il y a deux fois plus de livres que sur l'autre. Combien de livres y a-t-il sur chacun des rayons? » est traité de la façon suivante : 18 livres sur 2 rayons ... sur un il y a deux fois plus ... non ça ne marchera pas ... si on divise également il y aurait neuf ... mais il y a deux fois plus sur un rayon ... sur un neuf ... non égale neuf divisé par deux ... quatre et une demie ... et douze et une demie. Non quatre et sur l'autre quatorze ... Pourquoi quatre ... La moitié est impossible.

Avec les interventions de l'enseignant, cet adulte réussit à trouver la solution. L'enseignant lui donne un second problème tout à fait semblable au premier, 
mais en substituant " deux livres de plus » à « deux fois plus de livres ». L'adulte reproduit la solution qu'il a trouvée pour le premier problème.

d. Incapacité à effectuer des calculs simples : de nombreux exemples sont fournis.

Ces travaux montrent l'ancrage "médical » des études, ancrage bien compréhensible : population appréciable de blessés de guerre, foisonnement de recherches sur le fonctionnement cérébral. De tels travaux sont toujours réalisés. Nous pensons en particulier aux travaux réalisés par Pesenti, Deloche et Seron (Deloche et Seron (1987); Pesenti et Seron (2000)), travaux bien connus de plusieurs didacticiens. Ces travaux permettent de donner un sens aux erreurs des élèves. Plus encore, les défauts relevés sont toujours invoqués: défaut d'attention, hyperactivité, persévérance, défaut de planification. À cet effet, nous reproduisons la définition adoptée par le National Joint Committee for Learning Disabilities, en 1981 (voir Leong (1982, p. 5), traduction libre).

"Difficultés d'apprentissage est un terme générique qui réfêre à un groupe hétérogène de désordres qui se manifestent par des difficultés visibles dans l'acquisition et l'utilisation de l'écoute, de la parole, de la lecture, de l'écriture, du raisonnement ou de difficultés en mathématiques. Ces désordres sont intrinsèques à un individu et présumés être dus à un dysfonctionnement du système nerveux.»

Leong ajoute aussi que "les habiletés d'apprentissage et leurs désordres impliquent des processus différents, quoique reliés (ex. : écouter et parler (représentation phonétique); lire et écrire (représentations phonologiques, syntaxiques, sémantiques abstraites)) ». Cette vision prend appui sur les travaux en intelligence artificielle, en sciences cognitives, en psycho-linguistique. Inspirées par ces travaux, les études réalisées par Ginsburg (1977, pp. 124-125); pp. 140-143, traduction libre) ont été maintes fois citées par les chercheurs et les didacticiens. Ginsburg a conduit plusieurs entrevues auprès d'élèves présentant des difficultés en mathématiques. Plusieurs de ses observations et de ses interprétations sont relativement similaires à celles proposées antérieurement par Luria. Il ne s'agit pourtant pas de populations comparables. Les conduites et les interprétations suivantes montrent bien cette parenté : 


\begin{tabular}{|c|c|}
\hline $\begin{array}{l}23 \\
+ \\
5 \\
73\end{array}$ & \\
\hline $\begin{array}{l}19 \\
+ \\
16 \\
215\end{array}$ & $\begin{array}{l}1+1=2 \quad 9+6=15 \ldots \text { cet élève constate que } 215 \text { contient trop de chiffres... } \\
\text { II décide d'ignorer le } 5 \ldots \text { produisant la réponse } 21 . \\
15-7=65 \text { cet élève produit par ailleurs une réponse adéquate lorsque le calcul lui } \\
\text { est donné verbalement. Cet élève n'est pas affecté par des réponses contradictoires... } \\
\text { II croit qu'il est normal que nous obtenions des réponses différentes lorsque nous les } \\
\text { produisons verbalement ou par écrit. Ce fait, nous dit Faas (1980) a été pointé avec } \\
\text { beaucoup d'emphase par Werner. }\end{array}$ \\
\hline
\end{tabular}

\begin{tabular}{|c|c|}
\hline $\begin{array}{l}0 \\
+ \\
1 \\
20\end{array}$ & $\begin{array}{l}\text { Cet élève persiste à utiliser le même procédé lorsqu'il lui est demandé d'utiliser } \\
\text { une autre façon de faire le calcul, essayant de reproduire avec ses doigts le procédé } \\
\text { utilisé par écrit. Mais Ginsburg observe que le mot «plus » utilisé dans la } \\
\text { présentation de la consigne est déterminant. En effet, lorsqu'il demande à l'élève } \\
\text { « Combien font } 10 \text { et } 1 \text { ? », l'élève répond rapidement } 11 \text {. }\end{array}$ \\
\hline
\end{tabular}

Farhnam-Diggory (1979, p. 95, traduction libre) conclut ainsi sur ces similitudes entre les résultats obtenus par Ginsburg et ceux obtenus par Luria : «quelques formes de difficultés en arithmétique ou en lecture peuvent avoir une cause cérébrale ".

Ces interprétations montrent surtout une orientation du regard sur l'élève, abstraction faite de la position de cet élève dans le système didactique.

Comme le souligne Farhnam-Diggory, les études en sciences cognitives et en intelligence artificielle ont toutefois permis de raffiner les études comportementales sur les difficultés d'apprentissage; on parle ainsi de difficultés d'accéder à la mémoire sémantique et de difficultés dans la coordination d'informations. Un élève peut ne pas pouvoir nommer un nombre, non pas parce qu'il ignore le nom de ce nombre, mais parce qu'il ne peut rappeler ce nom assez rapidement. Un élève peut ne pas pouvoir additionner facilement les nombres 7 et 5, en ayant recours à un procédé de comptage avant, parce qu'il ne peut coordonner en mémoire de travail le comptage des nombres qu'il rappelle.

Si dans les études antérieures sur les relations entre l'intelligence (Wechsler (1974); Terman et Merrill (1960)) et le rendement scolaire, les épreuves de mémoire dans les tests d'intelligence occupaient une place importante (empan mnémonique), les capacités de mémorisation n'étaient pas utilisées pour examiner les difficultés des élèves en arithmétique. L'apport des théories sur la mémoire est donc de permettre de mieux lier certaines difficultés au fonctionnement de la mémoire.

L'analyse de procédés (ou de tâches), en terme de coût mnémonique, n'est pas négligeable. Si cette analyse poursuit des objectifs didactiques (et non exclusivement psychologiques), comme c'est le cas dans un grand nombre de travaux actuels en 
didactique des mathématiques, elle peut être utile à tous, en particulier aux élèves faibles.

Nous ne saurions passer sous silence les applications réductionnistes de la théorie de Piaget dans l'enseignement, notamment dans l'enseignement aux élèves en difficulté. Dans les années 70, les épreuves piagétiennes ont fait partie de la valise diagnostique de l'orthopédagogue et été à l'origine d'un travail important en enseignement et en rééducation. À ce moment, nous pouvions dire, sans hésiter, qu'il existait bel et bien un travail spécialisé auprès d'élèves en difficulté.

Il faut enfin reconnaître les travaux en intelligence artificielle sur le diagnostic des « bugs » en arithmétique (Brown et Burton (1978)). Ces travaux se situent dans le prolongement de plusieurs études sur les erreurs de calcul. En 1926, Buswell et Johns (voir Faas (1980, p. 263, traduction libre)) établissent une liste de 24 erreurs sur la soustraction; nous en reproduisons quelques-unes :

"Erreurs sur les combinaisons. Ne prend pas en compte le fait d'avoir emprunté. Erreurs dues à la présence de 0 dans le premier terme. Inversion des termes de la soustraction. Néglige d'emprunter, donnant 0 pour réponse. Erreur de lecture. Omet une colonne. Utilise un procédé d'addition essaierreur."

Les travaux de Brown et Burton ont permis de modéliser les règles productrices de ces erreurs et de plusieurs autres. Par la suite, dans leurs études sur les erreurs des élèves en division, Conne et Brun (1991) ont construit une interprétation de ces erreurs, montrant l'utilité de la notion de schème pour " voir autrement » les erreurs, pour les voir comme des produits d'un élève qui a « bel et bien » appris.

\section{Remédiation des difficultés d'apprentissage}

Durant cette première période, la remédiation des difficultés procède souvent par un ré-enseignement des objets de savoir, utilisant alors des modèles ou des dispositifs matériels et partant du schéma d'enseignement-apprentissage suivant : travail sur des modèles ou des dispositifs matérialisés, travail sur des dispositifs figuratifs, travail sur des dispositifs symboliques ou canoniques. On peut mettre en évidence un découpage des objets se traduisant souvent par un apprentissage des gestes à faire. Un tel découpage et un tel enseignement des gestes peuvent toujours être observés, comme le montrent plusieurs études récentes (nous y reviendrons). On assiste aussi à la mise en place d'un curriculum découlant d'une interprétation des travaux de Piaget sur les structures logiques, sur le nombre, interprétation bien documentée et coïncidant avec la réforme des «math modernes ». Citons l'ouvrage de Jaulin-Mannoni (1965) sur "La rééducation du raisonnement mathématique ", ouvrage qui a fait beaucoup de bruit. Jaulin-Mannoni propose diverses activités sur l'espace, le temps, le nombre, le symbolisme et la pensée mathématiques. Certaines de ces activités ont fait leur entrée dans les manuels scolaires pour les classes régulières. Dans l'introduction, on peut lire ce qui suit : "Le matériel concret n'est pas autre chose qu'un moyen de communication plus accessible à l'enfant que ne l'est la parole» (p. 15). Il s'agit d'une affirmation assez étonnante, que nous ne discuterons 
pas, mais qui supporte le recours souvent critiqué à des représentations composées de dessins d'objets et de signes opératoires.

Dans d'autres études, on suggère aussi un travail important sur le vocabulaire, prenant en compte le fait que plusieurs élèves qui présentent des difficultés ne savent interpréter et utiliser adéquatement le vocabulaire utilisé en mathématiques. Boehm (1970 : voir Faas (1980)) a ainsi proposé plus de 90 mots de ce vocabulaire. Bref, ce qui est fait est conséquent au diagnostic posé, et en accord avec les représentations de l'enseignement et de l'apprentissage. L'enseignement auprès d'élèves présentant des difficultés d'apprentissage repose souvent sur l'ingéniosité de l'enseignant. Cette ingéniosité peut être déployée d'autant plus aisément que les dispositifs d'enseignement sont peu élaborés, laissant à l'enseignant le choix de construire ses propres tâches et de les mener comme il l'entend.

Dans cette période, se développent divers outils conceptuels et méthodologiques visant à mieux comprendre les productions des élèves, à donner un sens à leurs productions : entrevue clinique, entrevue diagnostique, entrevue rééducative, analyse de protocoles. Plusieurs chercheurs canadiens, américains et européens ont contribué à ce travail.

\section{Seconde période : postérieure à 1980}

Au cours des vingt dernières années, le diagnostic des difficultés d'apprentissage en mathématiques a pratiquement cessé de reposer sur une batterie d'épreuves psychologiques, cognitives, pédagogiques et disciplinaires. Le peu d'intérêt didactique des résultats obtenus à la suite de l'administration de ces épreuves, la conviction partagée par plusieurs praticiens et chercheurs, à l'effet que les difficultés d'apprentissage ne sont pas une caractéristique immuable d'un individu (structurale), ont sans doute permis de transformer cette pratique. Parler de difficultés d'apprentissage sans parler de l'institution dans laquelle elles se révèlent, des critères et des caractéristiques du fonctionnement de cette institution, des enjeux pour les différents membres de cette institution, pose problème et limite l'action dans cette institution.

Les élèves dont le rendement scolaire est insuffisant, les élèves qui subissent des échecs scolaires, les élèves dits "faibles ", vivent, certes, une situation différente de celles des autres élèves de leurs classes respectives. Cette situation se rencontre dans les classes spéciales, mais aussi dans les classes régulières. Les enseignants de ces classes doivent composer avec cette situation.

Dans un premier temps, nous examinons les études visant à mieux comprendre ce qui pourrait différencier les élèves qui présentent « des difficultés » en mathématiques des autres élèves. Nous présentons, dans un second temps, certaines propositions didactiques visant à faciliter les apprentissages de ces élèves.

Depuis 1980, préoccupés par les difficultés des élèves en mathématiques, les chercheurs ont élaboré des outils conceptuels et méthodologiques pour identifier les connaissances et les représentations des élèves, dont les rapports aux objets du 
savoir enseigné sont jugés problématiques. Notre communauté de chercheurs québécois a grandement contribué à cette œuvre. Plusieurs chercheurs québécois ont ainsi procédé à des analyses conceptuelles des savoirs enseignés et des problèmes que soulève leur construction; plusieurs ont aussi puisé, dans les tâches et les problèmes présentés en classes, des informations qui leur ont permis de mieux interpréter les réponses des élèves; plusieurs ont également proposé diverses manières de prendre appui sur les connaissances des élèves, pour les questionner, les confronter, les enrichir. Citons, entre autres, les études réalisées par De Blois (1996); numération de position), par Rosar, Van Nieuwenhoven et Jonnaert (2001); fractions), par Deneau, Thivierge-Ayotte et Schmidt (2001); égalités arithmétiques).

Nous ne saurions rendre compte, dans ce texte, de l'ensemble des recherches sur les difficultés des élèves en mathématiques, cet ensemble incluant les recherches citées précédemment. Nous avons choisi de restreindre notre champ d'investigation en nous intéressant aux études portant sur la résolution de problèmes mathématiques.

\section{Les élèves " en difficulté ou faibles " en résolution de problèmes mathématiques}

Les élèves " en difficulté ou faibles » en résolution de problèmes mathématiques ont été scrutés par un nombre important de chercheurs. Et pour cause! Résoudre des problème est entrer dans une pratique mathématique reconnue.

\section{Analyse des difficultés}

Il n'est pas sans intérêt de rappeler que, de tout temps, comme le souligne Brown (1986), les enseignants ont présenté à leurs élèves des problèmes à résoudre. Plus encore, comme le montre Sarrazy (1996), les enseignants ont toujours été invités à soumettre à leurs élèves des problèmes comportant des données manquantes, à demander à leurs élèves de rédiger des problèmes et de représenter les données des problèmes. Quelle est alors la signification de l'insistance actuelle sur l'activité de résolution de problèmes?

L'originalité de l'orientation actuelle, déjà amorcée dans les années 80 , réside, selon nous, dans une utilisation originale des problèmes dans l'enseignement. Les problèmes ne sont plus des exercices que les enseignants donnent à leurs élèves, selon une vision applicationniste des connaissances enseignées, mais des problèmes que les élèves doivent résoudre pour construire de nouvelles connaissances. Ce n'est pas ainsi par hasard que l'activité de résolution de problèmes a été associée à une vision constructiviste de l'apprentissage, dans une interprétation, par ailleurs souvent discutable, des travaux de Piaget (Brun (1994)). Dans les théories actuelles en didactique des mathématiques, les problèmes acquièrent la fonction de donner un sens aux savoirs mathématiques qui sont enseignés et appris. La résolution de problèmes est ainsi une "activité mathématique " ou " une interaction d'un sujet avec un milieu propice aux pratiques mathématiciennes» (Conne (1999, p. 35)), pratiques référées à celles de la société des mathématiciens (Rouchier (1991)).

Chez les chercheurs en didactique des mathématiques, l'intérêt pour la résolution de problèmes doit aussi être contextualisé par un rappel d'événements majeurs 
qui leur ont permis de se construire des «outils pour étudier les conduites des élèves » et pour « intervenir sur ces conduites ».

La conjonction des travaux de Polya (1965) sur l'activité engagée dans la résolution de problèmes de mathématiques, des travaux de Krutetskii (1976) sur les habiletés en mathématiques des élèves, des travaux sur la résolution de problèmes mathématiques et l'enseignement des mathématiques (Ginsburg (1977); Schoenfeld (1985), (1987)), des travaux en intelligence artificielle sur les différences entre les solutionneurs experts et novices (Hinsley, Hayes et Simon (1978); Mayer (1981), (1985); Newell et Simon (1972); Riley, Greeno et Heller (1983)), des travaux sur la représentation (Anderson (1976); Kintsch (1977); Rumelhart et Ortony (1977); Mounoud (1985); Vergnaud (1981), (1983)), sur les stratégies et les processus cognitifs et métacognitifs (Mayer (1981), (1985); Richard (1990); Vygotsky (1978); Wong (1992)), a ainsi ouvert un champ, « encore fort actif ou en plein débroussaillage », aux chercheurs en didactique des mathématiques préoccupés par les difficultés des élèves en résolution de problèmes.

Pour montrer comment les résultats de ces recherches orientent l'analyse des difficultés des élèves, nous reproduisons quelques énoncés de problèmes proposés et analysés par Julo (1995).

\section{Énoncés A : (Julo (1995, p. 154))}

1 Tom et Anne ont 9 noisettes à eux deux. Trois de ces noisettes appartiennent à Tom. Le reste appartient à Anne. Combien Anne a-t-elle de noisettes?

2 Tom et Anne ont 9 noisettes à eux deux. Tom a trois noisettes. Combien Anne a-t-elle de noisettes?

Julo mentionne que les difficultés observées chez les élèves dans la résolution du $2^{\mathrm{e}}$ problème proviennent de l'incompréhension du fait que les trois noisettes qui appartiennent à Tom font partie de la collection de 9 noisettes. Plusieurs élèves choisissent alors d'additionner 9 et 3 .

\section{Énoncés B : (Julo (1995, p. 104))}

1 Une école de 75 élèves veut organiser une sortie. À ce moment de l'année, il y a dans la caisse de l'école $525 \mathrm{~F}$. De plus, le conseil municipal donne $20 \mathrm{~F}$ par élève. Pour le transport, il faut 2 cars et le prix de location d'un car est de 965F. La visite du zoo coûte 50 F. Y a-t-il assez d'argent pour la sortie?

2 Une école de 75 élèves veut organiser une sortie. À ce moment de l'année, il y a dans la caisse de l'école $4150 \mathrm{~F}$. De plus, le conseil municipal donne $20 \mathrm{~F}$ par élève. Pour le transport, il faut 2 cars et le prix de location d'un car est de 965 F. La visite du zoo coûte 50 F. Y a-t-il assez d'argent pour la sortie?

Julo souligne plusieurs difficultés que peuvent rencontrer les élèves dans la résolution de ces problèmes :

a. difficultés liées à la représentation : schéma " recettes/dépenses », connaissances du monde : sélection et organisation de toutes les informations pertinentes (ex : 20 F par élève... 75 élèves...); 
b. évaluation-validation de la solution réponse : problème 1 : si on calcule $50 \mathrm{~F}$ par élève, on n'a pas suffisamment d'argent; quoi faire? s'en tenir à cette réponse ou revenir au texte du problème pour une réinterprétation... des données...?

Dans cette étude, Julo (1995) montre également comment les élèves faibles sont sensibles aux indices ou aux consignes qui accompagnent la présentation des problèmes. Ce résultat rejoint ceux montrés dans plusieurs études, prenant en compte la notion de contrat didactique (Brousseau, 1998; Sensevy (1998)). Comment intervenir auprès de ces élèves?

\section{Propositions didactiques}

Plusieurs propositions didactiques émanent des travaux sur la résolution de problèmes mathématiques. Nous choisissons de présenter quelques propositions qui ont marqué la communauté des didacticiens et des chercheurs en didactique.

\section{a. enseignement de stratégies et d'heuristiques}

La connaissance des processus, stratégies et heuristiques en résolution de problèmes et des difficultés rencontrées par plusieurs élèves a conduit plusieurs chercheurs à proposer l'enseignement de ces processus, stratégies et heuristiques. Schoenfeld (1985), (1987) a réalisé plusieurs recherches sur l'enseignement des mathématiques privilégiant une approche qui met l'accent sur l'utilisation d'heuristiques en résolution de problèmes. Il formule ainsi son intention (1987, p. 18) :

"Mon intention était de poser la question des heuristiques de résolution de problèmes dans la perspective des études en sciences cognitives : Quel niveau de spécification doit-on atteindre pour décrire les stratégies de résolution de problèmes de manière à ce que les étudiants puissent les utiliser ».

Les résultats des expériences d'enseignement qu'il a réalisées sont probants. Les étudiants qui ont été entraînés à utiliser des heuristiques peuvent résoudre des problèmes similaires à ceux qu'ils ont résolus durant l'enseignement, mais aussi des problèmes qui ne sont pas similaires à ces derniers. Ces étudiants se montrent enfin capables de résoudre des problèmes complexes.

Les études réalisées par Schoenfeld concernaient des populations d'élèves ou d'étudiants ne présentant pas de difficultés graves d'apprentissage. Elles ont cependant inspiré nombre de chercheurs préoccupés par l'enseignement aux élèves présentant de telles difficultés. Des études similaires sur l'enseignement de stratégies cognitives et métacognitives sont toujours conduites. À titre d'exemple, on retrouve les recherches conduites par Montague (Montague (1992), (1997); Montague et alii (2000)). Dans ces recherches, l'enseignement des stratégies est organisé en leçons et son effet sur les performances des élèves en résolution de problèmes est examiné. Dans le programme Solve it, les stratégies enseignées sont les suivantes (Montague (1992, p. 234), traduction libre) : 


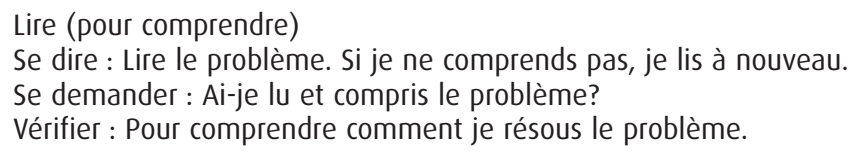

Si on ne peut douter des effets de cet enseignement, il est difficile d'aller au-delà de cette réception, ne connaissant généralement que des tâches proposées et les performances des élèves à ces tâches. Enfin, le fait que de telles recherches se poursuivent montre bien, il nous semble, la difficulté de penser des situations visant à engager les élèves dans une activité mathématique féconde.

\section{b. constructions d'environnements immédiats, disponibles et conditionnels visant à prévenir ou pallier les dysfonctionnements des élèves}

Dans son ouvrage, Julo (1995) fait aussi état de modalités visant à prévenir la multiplication des dysfonctionnements. Nous les résumons :

- Recourir à la multi-représentation (p. ex : problèmes similaires (contextes différents)). Si les effets sur les performances en résolution de problèmes sont positifs, il importe de se demander comment on peut y recourir, sans trop de risques de produire des effets de contrat (Brousseau (1998)) qui feraient illusion sur l'authenticité des connaissances imputées à l'élève. 
- Recourir à l'aide à la représentation :

1. entraîner les élèves à se représenter des classes de problèmes bien déterminées, en recourant aux travaux de Vergnaud (1981), (1983) sur les problèmes additifs et multiplicatifs, ou encore, aux travaux sur les schémas de problèmes (voir les travaux cités précédemment) : traduction graphique d'un énoncé. Les risques liés à l'utilisation de schémas de problèmes, ou d'une forme particulière de représentation, doivent être examinés. Vaut mieux «faire en sorte qu'un problème donné soit compris et résolu » (Julo (1995, p. 147))

2. définir un environnement: "... au point de départ, une problématique c'est-à-dire quelque chose d'abstrait qui associe un objet (de nature mathématique ...) et une tâche particulière. [...] L'objectif lorsqu'on fabrique un problème, est de transformer cette problématique en une situation de résolution de problèmes c'est-à-dire, en fait, un environnement avec lequel les élèves puissent interagir pour accéder à la problématique et la traiter. Il existe plusieurs façons de décrire et de modéliser un tel environnement: du point de vue de son contenu mathématique, [...], du point de vue des processus didactiques qui le caractérisent ou encore du point de vue du fonctionnement cognitif lié aux interactions qu'il permet, [...]» (Julo (1995, p. 148)). Julo distingue divers environnements : l'environnement immédiat du problème (données et autres éléments (matériel, consigne, etc.)); l'environnement disponible constitué de tous les éléments disponibles pour résoudre le problème, et qui ne sont pas présents dans l'environnement immédiat (aide que l'on peut solliciter; aides provenant de notes de cours, de fiches, etc.); l'environnement conditionnel (aide ponctuelle, interventions, ou tutorat). Il ajoute "nous avons toujours tendance à aider trop et [...] cette aide se situe, presque toujours, du côté des procédures et non du côté des représentations » (p. 152).

- Recourir à des tâches annexes : surtâches portant généralement sur le traitement des informations de l'énoncé (lire l'énoncé à voix haute, questions sur l'énoncé, etc.); un des risques est d'inscrire, en règles à suivre, les suggestions faites, ou d'ajouter d'autres tâches, ce qui alourdit souvent inutilement le travail.

\section{c. le travail sur l'erreur et le changement des habitudes de travail des élèves}

Nombre de chercheurs en didactique des mathématiques se sont préoccupés du traitement de l'erreur dans l'enseignement. Avant d'examiner quelques expériences didactiques, il nous semble important de reproduire les remarques effectuées par Chevallard (1988, p. 75) à la suite d'une étude de la copie d'un élève de terminale, lors d'une interrogation écrite :

"L'erreur de l'élève n'est donc que l'effet et le symptôme, dont la répétition engendrera peut-être une situation d'attribution d'échec, d'un rapport au calcul algébrique - notre objet $O$ - à très faible idonéité. On imagine que, pour supprimer des "étourderies" telle celle constatée plus haut, c'est un 
travail profond qui devrait être entamé; rien de moins que le remaniement du rapport de l'élève au calcul algébrique!»

Remanier le rapport des élèves à certains objets du savoir mathématique, en allant au-delà de la face publique de l'erreur, est donc un travail complexe. Reconstruire la biographie didactique d'un élève, comme l'ont bien montré Mercier (1992) et DeBlois (1996), peut s'avérer fort précieux et déboucher sur des interventions efficaces. Intégrer un travail sur l'erreur aux dispositifs usuels d'enseignement peut aussi amener une transformation non négligeable des rapports publics et privés des élèves aux objets mathématiques. Dans son ouvrage, Sensevy (1998, pp. 101108) parle ainsi de l'intérêt du travail sur l'erreur :

"L'intérêt du travail sur l'erreur pourrait donc en classe être double :

- favoriser une activité de type épistémologique, que fournirait le fait de travailler sur ses propres erreurs ou les erreurs des autres. Ce travail serait aussi une tâche d'apprentissage;

- mettre à mal l'allant-de-soi de l'épistémologie quotidienne, aussi bien affectifs ("je suis l'erreur que j'ai commise») qu'épistémologiques ("le maître seul possède ontologiquement le pouvoir de repérer les erreurs "), ces allant-de-soi pouvant s'apprécier comme nouveaux avatars de la position d'attente.

$[\ldots]$

Notre intention était de faire en sorte que l'élève construise un rapport à l'erreur désigné de manière déclarative aux élèves de la façon suivante :

Une erreur n'est pas une faute. Il n'y a pas à se sentir coupable de faire une erreur. On peut beaucoup apprendre de ses propres erreurs, ou des erreurs des autres. Il est important de savoir travailler sur ses (des) erreurs; il faut donc apprendre à le faire. Il est important de retenir certaines erreurs " types », qui vont servir de guide pour les futurs travaux. "

Dans le but de provoquer une rupture des habitudes des élèves dans le traitement de problèmes multiplicatifs, Sensevy invite les élèves à fabriquer des problèmes. Les élèves se pratiquent d'abord à reconnaître les différents cas de la typologie de problèmes définie par Vergnaud (1991). Divers énoncés de problèmes sur la soustraction leur sont soumis pour introduire cette typologie et les élèves doivent produire par la suite une fiche comportant 1 problème pour chacun des cas de la typologie, en inscrivant au verso de la fiche l'attribution de cas dans la typologie (correction). Chaque fiche est alors examinée par une dyade d'élèves. Si elle est jugée adéquate, elle est placée dans un fichier. Dans le cas contraire, la fiche est transformée, puis placée dans le fichier. En cas de désaccord persistant, l'enseignant intervient : "Le consensus sur la base duquel enseignant et élèves travaillaient était que tout au long du processus (fabrication-correction-vérification de la correctionchoix de la fiche-discussion en cas de désaccord), l'enseignant devait intervenir le 
moins possible.» (Sensevy (1998, p. 118)). Le repérage de l'erreur tombe sous la responsabilité de l'élève, plutôt que sur celle de l'enseignant. Dans cette perspective, la régulation ne se faisait pas "par les résultats attendus, mais par l'erreur nécessairement détectée et traitée par les élèves» (Sensevy (1998, p. 118); voir aussi Amigues (1994, p. 97)).

Ce travail a contribué à mettre en place des rapports à des objets nouveaux, à créer les habitus d'une pratique nouvelle. C'est dans ce cadre, qu'a succédé par la suite $\left(2^{\mathrm{e}}\right.$ année du projet), le travail sur la production de problèmes de fractions. Les problèmes ainsi construits par les élèves devaient respecter certaines consignes ayant trait à la typologie (p. ex : état final, transformation, etc.) et amener les élèves à " produire du nouveau et à prendre conscience des insuffisances de leurs énoncés " (Sensevy (1998, p. 121)).

Sensevy montre l'intérêt de ce travail dans la transformation des habitudes des élèves, dans leur prise de conscience de leur responsabilité à l'égard de la production d'erreurs, dans leur prise de conscience de leurs possibilités d'apprentissage, et dans les apprentissages réalisés. Un travail similaire de rédaction de problèmes multiplicatifs a aussi été mené avec succès auprès d'élèves faibles de milieux favorisés et défavorisés par Lemoyne, Giroux et Biron (1990).

\section{Conclusion}

Les élèves en difficultés d'apprentissage, comme nous l'avons évoqué précédemment, et comme le montrent plusieurs études (Sensevy (1998); PerrinGlorian (1993)), semblent souvent s'être installés dans une " position d'attente » qui "limite considérablement le désir, l'appétence» (Sensevy (1998, p. 61)). La rencontre avec l'ignorance qui devrait susciter le désir d'apprendre est souvent une rencontre ratée. De plus, même si on parvient à établir une biographie didactique (Mercier (1992)), même en comptant avec les mémoires didactiques (Brousseau et Centeno (1991)), même en recourant à des techniques variées visant à mieux documenter la mémoire personnelle de ces élèves (Coppé (1995)), il est difficile d'engager un dialogue didactique avec ces élèves. Encore plus que les autres, ces élèves ont un pouvoir certain sur l'avancée du temps didactique; ces élèves participent bel et bien à l'enseignement (Mercier (1998)). Nos expériences en classes d'adaptation nous montrent que les élèves disposent de nombreux moyens pour provoquer des pauses didactiques: passivité, nombreux appels à l'aide, travail effectué à la hâte, indiscipline, etc. Il importe de reconnaître les difficultés qu'éprouvent un grand nombre d'enseignants en adaptation scolaire. Nous ouvrons donc une fenêtre sur ces difficultés.

\section{Les enseignants en difficulté d'enseignement : ce qui les différencie des autres}

Un enseignement spécialisé se reconnaît d'abord par une extension du temps didactique. À cet égard, le travail réalisé par Favre (1993), (1997), sur l'enseignement 
en classes régulières et spéciales, est fort précieux pour montrer qu'il s'agit bel et bien de deux institutions différentes. Son travail sur l'enseignement de la multiplication montre aussi qu'il est possible d'agir dans et sur ces institutions. Mais avant d'explorer des façons d'agir, il est important de " parler un peu des enseignants en difficulté d'enseignement ».

Un enseignant est en difficulté d'enseignement lorsque les dispositifs qu'il croyait "pertinents " montrent leur inefficacité à produire des savoirs institutionnellement reconnus chez ses élèves. Conne et Favre (1999) ont bien montré cette difficulté.

Que faire alors? Avant de présenter ce qui pourrait être fait, il nous semble important de prendre quelques instants pour montrer que certains refuges des enseignants sont tout à fait compréhensibles, et trop souvent amplifiés par des applications de recherches servant de phares idéologiques. Nous avons pu montrer comment les recherches sur la résolution de problèmes et sur les erreurs de calculs ont débouché sur des pratiques "spéciales ». Les règles productrices des erreurs de calcul ont été interprétées comme s'il s'agissait des règles des élèves, des connaissances lacunaires des élèves, et non comme des produits d'apprentissage dénotant d'authentiques efforts de construction de connaissances chez les élèves (Conne et Brun (1991)). Mais regardons de plus près les pratiques en enseignement spécialisé.

Si dans l'enseignement spécialisé, plusieurs enseignants prolongent les prérequis ou effectuent un dressage des gestes (voir, entre autres, les études de Mercier sur les fractions (Mercier (1995)), rendant visibles tous les gestes producteurs des réponses attendues, il nous faut prendre acte de ce fait et avouer les difficultés d'enseignement. S'agit-il, comme aimeraient le croire les magiciens des théories d'apprentissage et d'enseignement, d'une application "behaviorale "? Nous n'en sommes pas si sûres. À cet effet, nous aimerions rappeler une expérience "spécialisée » et récente auprès d'un élève de $6^{\mathrm{e}}$ année.

Le travail clinique réalisé avec cet élève a été marqué par des actions souvent improvisées, témoignant de nos difficultés d'enseignement. Cet élève, dont les connaissances langagières et les performances en français étaient nettement supérieures à celles de son groupe de référence, a montré, dès notre première rencontre, un désinvestissement, voire une indifférence marquée pour les mathématiques. Avouant produire des erreurs en multiplication, nous lui proposons les calculs suivants: 239 x 452; 240 x 452; 2400 x $4520 ; 2390$ x 452; nous lui demandons de faire le moins de calcul possible, mais de s'assurer qu'il a bien obtenu les réponses justes. Parfait, nous dit-il. Et il fait tous les calculs, commettant alors plusieurs erreurs. Nous poursuivons en lui demandant de faire : 24000 x 40 001; il applique, une fois de plus, l'algorithme usuel, application erronée, compte tenu du nombre de rangées comportant un alignement de 0 qu'il doit produire. Enfin, en désespoir de cause, nous lui demandons s'il peut composer un énoncé de problème comportant les nombres des deux premiers calculs et partir de la réponse obtenue à un des problèmes, pour trouver la réponse à l'autre problème. Les problèmes rédigés sont d'une pauvreté contextuelle désarmante, mais sensés et, oh! Miracle!, il trouve facilement comment transformer la réponse au premier problème pour produire la 
réponse au second problème. Mais il avoue préférer faire les calculs qu'on lui demande. À la fin de cette rencontre, il nous demande de lui faire faire des problèmes à la seconde rencontre car, dit-il, il doit passer un examen d'étape. Nous choisissons des problèmes voisins de ceux rencontrés dans son manuel. Les performances de l'élève sont très faibles; les erreurs commises sont voisines de celles décrites dans les études. Voici quelques-uns des problèmes présentés à l'élève :

1. Dans la classe de Peter, $1 / 4$ des élèves préfèrent le basketball; $3 / 10$, le hockey; $16 \%$, le baseball. Le reste des élèves préfèrent le patin. Quel pourcentage des élèves préfèrent le patin?

2. Nicolas a 36 pièces de monnaie. Les $5 / 6$ de ses pièces sont des pièces de $25 \mathrm{c}$. Le reste de ses pièces sont des pièces de 1c. Combien de pièces de 1c Nicolas a-t-il?

3. Lorsqu'on a demandé à Fred l'âge de ses sœurs, il a répondu : Lise a le tiers de l'âge d'Annie, France a 4 ans de plus que Lise et la somme de leurs âges est de 44 ans. Pouvez-vous déterminer les âges respectifs de chacune des sœurs de Fred?

4. Un commerçant très maniaque a l'habitude de compter le nombre de raisins qu'il a sur l'étalage de la devanture de son commerce. Aujourd'hui, il a réussi à compter 234468 raisins. Par un curieux hasard, 117 clients se présentent en même temps et souhaitent se partager tous les raisins. Pensez-vous que chacun des clients peut avoir au moins 10000 raisins? Au moins, 1 000? Au moins, 2000 ? Êtes-vous capable de trouver le nombre de raisins de chacun, en faisant très peu de calculs? Et si le commerçant trouve encore dans sa réserve 2457 raisins, avec combien de raisins les clients pourront-ils partir?

Cette situation survient au moment où nous examinons le travail de Sensevy (1998) sur la fabrication de problèmes. Nous nous remémorons, à cette occasion, les travaux de l'équipe de Vergnaud dont fait état Rouchier (1991), Rouchier (1999). Nous décidons de donc de reproduire la typologie de Vergnaud (1981), (1983) sur les problèmes additifs et multiplicatifs. Nous demandons à l'élève d'étudier cette typologie et de prendre tous les problèmes qui apparaissent dans les épreuves de fin d'étape, de les rassembler selon cette typologie. Nous lui proposons aussi divers schémas pouvant lui être utiles pour organiser les données des problèmes qu'il juge particulièrement difficiles. Nous insistons pour qu'il laisse de côté les problèmes qui lui semblent faciles. À titre d'exemple, voici les schémas proposés pour un des problèmes multiplicatifs : J'ai 9 paquets de gommes. Chaque paquet contient le même nombre de gommes. Dans les 9 paquets, j'ai 108 gommes. Combien de gommes devraisje avoir si au lieu de 9 paquets j'avais 27 paquets. 


\begin{tabular}{|c|c|}
\hline \multicolumn{2}{|c|}{ Schémas : \#1 tableau } \\
\hline Paquets & Gommes \\
\hline 9 & 108 \\
\hline 27 & $?$ \\
\hline
\end{tabular}

Paquets

9

27
Schémas : \#2 flèche

$\begin{array}{cc} & \text { Gommes } \\ \rightarrow & 108 \\ \rightarrow & ?\end{array}$

L'élève rassemble presque sans erreurs les problèmes puisés dans les épreuves de fin d'étape, en recourant à la typologie. Il constate que, si on fait abstraction des contextes et des nombres utilisés, les problèmes « se laissent avaler - comme il dit par ces choses ». Et les outils «typologie » et « schémas » sont ensuite utilisés avec profit par cet élève, puis négligés lorsqu'ils sont intégrés. L'élève se construit enfin un outil «hybride " pour les problèmes qu'ils jugent plus difficiles, par exemple, pour le problème suivant: Nicolas a 36 pièces de monnaie. Les $5 / 6$ de ses pièces sont des pièces de 25c. Le reste de ses pièces sont des pièces de $1 \mathrm{c}$. Combien de pièces de $1 \mathrm{c}$ Nicolas $a-t-i l$ ?

\begin{tabular}{|c|c|c|c|c|}
\hline Pièces de 25c & $\rightarrow$ & Pièces en tout & $\rightarrow$ & Pièces de 1c \\
\hline$?$ & $5 / 6$ de & 36 & de $1 / 6$ & $?$ \\
\hline & & & & $? ? ? ? ?$ \\
\hline & & & & $\mathrm{R}: 6$ \\
\hline
\end{tabular}

La décision de proposer une typologie et des schémas de problèmes, décision prise presque en désespoir de cause, s'avère fructueuse. Parmi les problèmes jugés difficiles par l'élève, on retrouve ceux dont la solution fait appel à des calculs sur des grands nombres entiers, sur des nombres décimaux et sur des fractions. Nous nous sommes donc intéressées aux difficultés de l'élève en calcul arithmétique et sur les fractions. Dans un premier temps, nous avons eu recours à un couplage problème-calcul. Nous avons, par exemple, produit deux versions d'un des problèmes présentés dans le manuel de l'élève.

\section{Problème 1 :}

Un commerçant très maniaque a l'habitude de compter le nombre de raisins qu'il a sur l'étalage de la devanture de son commerce. Aujourd'hui, il a réussi à compter 234468 raisins. Par un curieux hasard, 117 clients se présentent en même temps et souhaitent se partager tous les raisins. Pensez-vous que chacun des clients peut avoir au moins 10000 raisins? Au moins, 1000 ? Au moins, 2 000? Combien de raisins chacun des clients pourra-t-il obtenir? 


\section{Problème 2 :}

Un commerçant très maniaque a l'habitude de compter le nombre de raisins qu'il a sur l'étalage de la devanture de son commerce. Aujourd'hui, il a réussi à compter 190304 raisins. Par un curieux hasard, 905 clients se présentent en même temps et souhaitent se partager tous les raisins. Pensez-vous que chacun des clients peut avoir au moins 10000 raisins? Au moins, 1 000? Au moins, 100? Combien de raisins chacun des clients pourra-t-il obtenir?

L'élève répond correctement aux trois premières questions de ces problèmes, mais ne peut effectuer correctement les calculs pour répondre à la dernière question. Ainsi, dans le second problème, il sait que chacun des clients pourra recevoir un nombre de raisins dans les centaines (expression utilisée par l'élève); il ne sait, par ailleurs, utiliser l'information précédente pour questionner le nombre 21,2, quotient de la division qu'il effectue. Prenant la décision de ne pas traiter directement cette erreur, nous avons recours à un enseignement de gestes sans parole, demandant alors à cet élève de produire la parole; cette démarche est souvent celle de l'enseignant qui commente, par exemple, les écritures qu'il produit au tableau. Nous effectuons donc des multiplications et des divisions d'entiers, de décimaux, de fractions. Et cette intervention s'avère pertinente. Il apparaît important de souligner que l'enseignement de gestes n'est pas en soi un enseignement à condamner; il faut comprendre la situation qui le porte.

Les histoires précédentes ne sont pas uniques. Elles montrent bien que les pratiques des enseignants en difficulté sont quelquefois des pratiques par défaut; leur issue semble plus incertaine que ne le sont les pratiques faisant partie de la boîte didactique de l'enseignant. Et pourtant! Comment agir sur les pratiques en enseignement spécialisé, voire en enseignement régulier?

Comme le montrent enfin plusieurs études, lorsque nous présentons un projet d'enseignement à des enseignants spécialisés, nous faisons souvent face à des déclarations sur la non adaptation de ces situations à leur clientèle d'élèves (Favre (1997); Gaudreau et alii (2001)). Cette réaction est tout à fait intelligible, si on prend en considération les pratiques courantes souvent renforcées par des discours sur l'importance de faire vivre des succès aux élèves présentant des difficultés, de procéder par étapes, etc. Cette réaction est aussi rencontrée dans le milieu de l'enseignement régulier, mais soutenue par d'autres discours.

Quiconque parcourt les manuels en usage doit aussi reconnaître que des intuitions ou des idées ingénieuses sont peu ou mal exploitées dans l'enseignement, à défaut d'une problématisation des situations qui pourrait permettre, par des activités mathématiques pertinentes, une entrée dans des pratiques mathématiciennes (Conne (1999)). Parler de la problématisation de l'enseignement des mathématiques est aussi parler de connaissances et de savoirs (Brousseau (1998); Conne (1996); Rouchier (1991)). Dans l'enseignement spécialisé, qui veut partir des connaissances des élèves doit souvent faire acte de devin, à moins de s'en tenir à une analyse de 
surface des conduites des élèves. Par ailleurs, dans un enseignement problématisé, ces connaissances agissent et se montrent assez fréquemment à l'observateur curieux.

\section{Les attentes et les pratiques en classes spéciales et régulières des futurs enseignants en adaptation scolaire}

Intervenir efficacement auprès d'élèves présentant des difficultés d'apprentissage est le souhait de tout enseignant en adaptation scolaire. $\mathrm{Si}$, au fil des ans, les enseignants en exercice et les chercheurs en didactique des mathématiques, ont construit et éprouvé un certain nombre d'interventions efficaces, il en est autrement chez les futurs enseignants en adaptation scolaire. Préparer ces futurs enseignants à intervenir efficacement s'avère une entreprise complexe. Les attentes et les pratiques en classes spéciales et régulières des enseignants en exercice, comme nous en avons brièvement fait état, sont souvent différentes de celles prônées par les didacticiens et les chercheurs en didactique des mathématiques. Les professeurs impliqués dans les programmes de formation des enseignants en adaptation scolaire ne peuvent faire abstraction de ces différences. Ils se doivent d'agir auprès des futurs enseignants pour les convaincre de la nécessité de problématiser l'enseignement auprès d'élèves présentant des difficultés d'apprentissage.

Dans cette perspective, nous avons l'habitude de demander, aux futurs enseignants en adaptation scolaire, de se prononcer sur la pertinence de situations d'enseignement. Nous avons ainsi présenté, à des étudiants de $2^{\mathrm{e}}$ et de $3^{\mathrm{e}}$ année inscrits dans le programme de formation en adaptation scolaire, la situation suivante sur les fractions, en leur demandant: a) de faire les tâches demandées; b) de se prononcer sur l'utilisation de ces tâches : 1- en classe spéciale et pour les élèves de quelle année scolaire; 2 - en classe régulière et pour les élèves de quelle année scolaire. Ce travail a été réalisé en début d'année, lors du $2^{\mathrm{e}}$ cours (années académiques 1995-96 et 1996-97). Ce sont les mêmes étudiants qui ont effectué ce travail deux années consécutives.

Le choix des étudiants de $2^{\mathrm{e}}$ et de $3^{\mathrm{e}}$ est motivé par les raisons suivantes : a) les étudiants de $2^{\mathrm{e}}$ n'ont pas encore effectué un stage en classe spéciale et entreprennent leur premier cours sur les difficultés d'enseignement et d'apprentissage des mathématiques; b) les étudiants de $3^{\mathrm{e}}$ entreprennent leur troisième cours sur les difficultés d'enseignement et d'apprentissage des mathématiques et ont eu l'occasion de réaliser un stage en classe spéciale.

La situation comprend 5 parties. Nous donnons un bref aperçu des tâches qu'elles comportent. Dans la réalisation de ces tâches, les élèves sont répartis en équipes (2 à 3 élèves par équipe). 
Partie 1 : Représentations des fractions $1 / 2,1 / 4$ et $1 / 8$, sur une feuille non lignée ou quadrillée : pliage, partage; la feuille constitue le tout Matériel :

des feuilles 8 1/2 par 11 (pouces), non lignées $(21,6 \mathrm{~cm}$ par 28,6 $\mathrm{cm}$ : mesures approximatives).

Consigne 1 :

L'enseignant donne une feuille à chacune des équipes et leur demande : a) de plier une feuille en 2 et d'indiquer quelle fraction représente chacune des parties; b) de continuer à plier la feuille précédente en 2 et d'indiquer quelle fraction représente chacune des parties; c) de continuer à plier la feuille précédente en 2 et d'indiquer quelle fraction représente chacune des parties; d) d'indiquer quelle fraction représenterait chacune des parties, si on pliait encore en 2 la feuille précédente; e) de prévoir s'il est possible d'obtenir 1/100 d'une feuille, en continuant le pliage.

Consigne 2:

L'enseignant reprend la feuille pliée et donne une nouvelle feuille à chacune des équipes, en leur demande de partager cette feuille pour montrer successivement les fractions $1 / 2,1 / 4,1 / 8,1 / 16$. Il indique que la feuille constitue le tout à partager. Il ajoute qu'ils ne peuvent plier la feuille; ils peuvent utiliser l'une ou l'autre des règles qu'ils possèdent. Il précise que lorsqu'ils auront terminé, ils pourront comparer les parties obtenues avec celles qu'ils ont obtenues par pliages; ils pourront ainsi s'ils le souhaitent modifier (ou recommencer) les partages.

Ces étapes terminées, l'enseignant examine avec les élèves leurs réponses et leur demande s'il est plus facile d'obtenir des représentations plus justes par pliages ou par partages et pourquoi; il leur demande aussi comment ils pourraient obtenir de meilleurs résultats dans chacun des cas.

Partie 2 : Représentations des fractions $1 / 2,1 / 4$ et $1 / 8$, sur une feuille quadrillée : pliage, partage; la feuille constitue le tout

Les tâches présentées dans la partie 1 sont reprises. À la fin de cette activité, l'enseignant leur demande si l'utilisation de feuilles quadrillées permet d'obtenir des représentations plus justes des fractions que l'utilisation de feuilles non quadrillées et pourquoi.

Partie 3 : Représentations des fractions $1 / 2,1 / 4$ et $1 / 8$ sur une feuille quadrillée (ou non) : partage

L'enseignant demande maintenant à chacune des équipes de représenter sur une feuille quadrillée ou non, la décision leur appartenant, les fractions précédentes. Il indique qu'ils ne sont pas tenus d'utiliser la feuille entière; ils doivent essayer de se rapprocher le plus près possible du tout qui pourrait correspondre à la feuille entière. 
Après examen des réponses en groupe, les élèves sont invités à indiquer s'il est plus facile d'obtenir des représentations plus justes dans cette situation que dans celles des parties 1 et 2 et à expliquer pourquoi. À la fin de cette partie, les élèves sont invités à trouver ensemble des moyens pour représenter, de la manière la plus juste, des fractions sur papier.

\section{Partie 4 : Représentations des fractions $1 / 3,1 / 6$ et $1 / 8$ sur une feuille quadrillée (ou non)}

Cette partie est similaire à la partie 3; seules les fractions diffèrent.

\section{Partie 5 : Représentations des fractions $1 / 3,1 / 4$ et $4 / 10$ sur une feuille quadrillée (ou non)}

Cette partie est similaire à la précédente, seules les fractions diffèrent.

Le tableau suivant fait état des choix des étudiants, en ce qui concerne chacune des tâches que comporte la situation précédente. Pour chacune des années du baccalauréat, les choix des étudiants sont tout à fait comparables; quelques étudiants seulement ne partagent pas l'opinion des autres étudiants de leurs groupes respectifs. On remarque, par ailleurs, entre les étudiants de $2^{\mathrm{e}}$ et de $3^{\mathrm{e}}$ année, une transformation des choix selon la clientèle d'élèves en classes spéciales. Le stage en classe spéciale, effectué au cours de la $2^{\mathrm{e}}$ année, semble avoir affecté ces choix, compte tenu du fait que l'enseignement des fractions ne faisait pas partie du cours de la $2^{\mathrm{e}}$ année en didactique des mathématiques. Certains étudiants s'appuient à la fois sur les pratiques rencontrées durant leur stage et sur les difficultés de ces tâches, difficultés éprouvées lors de leur réalisation, pour soutenir leurs choix. Les tâches associées aux $4^{\mathrm{e}}$ et $5^{\mathrm{e}}$ parties de la situation sont généralement celles qui sont jugées trop complexes; le commentaire souvent donné est le suivant : absence d'un facteur commun pour les dénominateurs. Un grand nombre d'étudiants dessinent un carré dont le côté correspond au produit des dénominateurs. 
Les rencontres singulières entre les élèves présentant

\begin{tabular}{|c|c|c|c|c|}
\hline \multirow[t]{2}{*}{ Tâches } & \multicolumn{2}{|c|}{ Classes spéciales } & \multicolumn{2}{|c|}{ Classes régulières } \\
\hline & $\begin{array}{c}\text { Baccalauréat } 2 \\
\text { (88 des } 92 \text { étudiants) }\end{array}$ & $\begin{array}{c}\text { Baccalauréat } 3 \\
\text { (71 des } 74 \text { étudiants) }\end{array}$ & $\begin{array}{l}\text { Baccalauréat } 2 \\
\text { (89 des } 92 \text { étudiants) }\end{array}$ & $\begin{array}{c}\text { Baccalauréat } 3 \\
\text { (73 des } 74 \text { étudiants) }\end{array}$ \\
\hline $\begin{array}{l}\text { 1a - pliage } \\
1 / 2-1 / 4-1 / 8\end{array}$ & $4^{e}-5^{e}$ & $5^{e}-6^{e}$ & $4^{e}-5^{e}$ & $4^{e}-5^{e}$ \\
\hline 1b - partage & $5^{e}$ & $6^{e}-\sec -1$ & $5^{e}-6^{e}$ & $6^{e}$ \\
\hline Prévision 1/100 & $6^{e}$ & sec- 1 ou 2 & $6^{e}$ & $6^{e}$ \\
\hline $\begin{array}{l}\text { 2a - quadrillée } \\
\text { pliage }\end{array}$ & $4^{e}-5^{e}$ & $5^{e}-6^{e}$ & $4^{e}-5^{e}$ & $4^{e}-5^{e}$ \\
\hline $\begin{array}{l}\text { 2b - quadrillée } \\
\text { partage }\end{array}$ & $5^{e}$ & $6^{e}-\sec -1$ & $5^{e}-6^{e}$ & $6^{e}$ \\
\hline 3- tout à définir & $5^{e}-6^{e}$ & $6^{e}-\sec -1$ & $5^{e}-6^{e}$ & $6^{e}-\sec -1$ \\
\hline $\begin{array}{l}\text { 4- tout à définir } \\
1 / 3-1 / 6-1 / 8\end{array}$ & $6^{e}-\sec -1$ & sec-1 - sec-2 & $6^{e}$ & $6^{e}-\sec -1$ \\
\hline $\begin{array}{l}5 \text { - tout à définir } \\
1 / 3-1 / 4-4 / 10\end{array}$ & sec-1 & $\sec -2$ & $6^{e}-\sec -1$ & sec-1 \\
\hline
\end{tabular}

Comme nous l'avons dit antérieurement, nous avons aussi demandé aux étudiants de réaliser chacune des tâches. Dans une des tâches de la partie 1, on demandait s'il était possible d'obtenir 1/100 d'une feuille en continuant le pliage par 2. Les étudiants de $2^{\mathrm{e}}$ et de $3^{\mathrm{e}}$ année, il faut le mentionner, n'avaient pas reçu un enseignement sur la didactique des fractions. 67 des 92 étudiants de $2 \mathrm{e}$ année et 51 des 74 étudiants de $3^{\mathrm{e}}$ année répondent oui, en ajoutant que 100 est un multiple de 2. Les autres étudiants qui répondent non, à chacune des années, procèdent à une énumération des parties obtenues, soit 2, 4, 8, 16, 32, 64, 128. Quelques étudiants ajoutent: $1 / 21 \quad 1 / 22 \quad 1 / 23 \quad 1 / 24 \quad \ldots$

Les résultats de l'enquête précédente ont été communiqués à tous les étudiants de $3^{\mathrm{e}}$ année. Les étudiants ont ensuite été invités à utiliser cette situation, lors de leur stage en adaptation scolaire, et à nous communiquer les résultats de cette application. Plusieurs étudiants nous ont alors fait part de leur étonnement à l'égard des connaissances utilisées avec profit par les élèves présentant des difficultés. Mettre en place les conditions pour provoquer un tel étonnement nous semble crucial, si nous souhaitons transformer les perceptions sur l'enseignement et l'apprentissage en classes spéciales.

\section{Conclusions}

Les rencontres entre les élèves présentant des difficultés d'apprentissage en mathématiques et leurs enseignants sont, depuis plusieurs années, jugées singulières 
tant par les chercheurs que par les praticiens. Les élèves présentant des difficultés d'apprentissage en mathématiques, dans nos institutions scolaires, ne peuvent passer inaperçus. Comme nous l'avons montré, aux premières investigations liant les difficultés d'apprentissage des élèves aux caractéristiques fonctionnelles et structurales de ces élèves (période antérieure à 1980), ont succédé des investigations prenant également en compte les difficultés de construction des savoirs mathématiques, le fonctionnement du système didactique, les programmes d'études, le matériel didactique, les situations et les contextes d'enseignement et d'apprentissage (période postérieure à 1980). Ces dernières investigations ont débouché sur des propositions et des interventions didactiques qui invitent à des transformations des pratiques d'enseignement et d'apprentissage.

L'enseignement auprès d'élèves présentant des difficultés d'apprentissage, comme le montrent plusieurs des études dont nous avons fait écho dans ce texte, ne peut aussi ignorer l'histoire scolaire de ces élèves. Tenir compte de l'histoire scolaire des élèves n'est pas chose facile. Cela peut conduire à un recyclage ou à une reprise de situations ou de tâches, reprise encombrée d'un nombre encore plus important d'ostensifs (gestes, discours, graphiques, etc.). Cela peut aussi, heureusement, conduire à une identification de connaissances non négligeables chez les élèves, connaissances souvent liées à des tâches et situations spécifiques. Dans une classe, les compétences et les connaissances des élèves peuvent alors devenir objets d'explicitation, de questionnement, de confrontation. De telles pratiques rejoignent celles mises de l'avant dans le programme actuel de formation de l'école québécoise, dans le programme d'études en mathématiques, et dans le plan d'action en matière d'adaptation scolaire (Publications du Ministère de l'éducation (2001) et (2003); http://www.meq.gouv.qc.ca/DGFJ).

$\mathrm{Si}$, comme nous l'avons montré, les chercheurs en didactique des mathématiques disposent maintenant d'outils conceptuels et méthodologiques leur permettant d'élaborer, avec les enseignants, des situations offrant aux élèves en difficulté des occasions de transformer leurs rapports aux mathématiques, de mieux apprendre les mathématiques, ils sont souvent, comme le sont les enseignants, démunis à l'égard de certaines conduites d'élèves. Il nous est ainsi apparu fondamental de souligner les difficultés que rencontre l'enseignant en adaptation scolaire. À notre connaissance, peu d'études ont porté sur les difficultés d'enseignement.

Les élèves qui présentent des difficultés d'apprentissage ont construit, au fil des ans, des habitudes de travail et d'étude, qui leur sont souvent néfastes. Comment aider les élèves à rompre ces habitudes, à adapter des conduites plus propices? Ces questions ont été objet de discussions lors des dernières journées Inter-IREM qui ont eu lieu à Dijon (24-25 mai 2002). Ces discussions, auxquelles ont pris part plusieurs chercheurs et enseignants français, ont permis d'apprécier la complexité de ces questions et plus encore, des réponses envisageables ou à explorer.

Nombre de questions, sur les difficultés d'apprentissage en mathématiques de plusieurs élèves, sur les dispositifs à mettre en place pour faciliter les apprentissages de ces élèves, sur les problèmes auxquels font face les enseignants en adaptation scolaire, demeurent sans réponse. La formation des enseignants en adaptation 
scolaire soulève également des questions importantes. À cet effet, nous avons donné un bref compte rendu d'une expérience visant à cerner l'évolution des attentes et des pratiques en classes régulières et spéciales d'un groupe de futurs enseignants en adaptation scolaire. Les résultats invitent à un dialogue soutenu entre les milieux de pratiques et les milieux de formation, entre les enseignants en adaptation scolaire et les professeurs responsables de la formation en didactique des mathématiques.

Pour conclure, il nous semble pertinent de résumer, en quelques mots, les changements de points de vue qui ont marqué la recherche et les pratiques en adaptation scolaire, au cours des sept dernières décennies. Pendant plusieurs décennies, les chercheurs ont cherché à mieux comprendre le fonctionnement des élèves présentant des difficultés d'apprentissage, élèves dits souvent à risque. On reconnaît aujourd'hui que ces élèves sont souvent, dans les institutions scolaires, placés en situation de risque. À cet effet, il nous semble approprié de rapporter certaines des questions formulées par Franklin (2000, p. 5) : «Instead of wondering, ... Is the child ready for school? And, is the child at risk of failing school?. ... Educators must also ask : Is the school ready for the child? And, Is the school at risk of failing the child? »

\section{Références bibliographiques}

Amigues, R. (1994). Construction des faits d'enseignement et voies de recherche en psychologie de l'éducation. Un exemple, les technologies éducatives, Habilitation à diriger des recherches, Université de Provence, Aix-Marseille 1.

Anderson, J.R. (1976). Language, Memory and Thought. Hillsdale, N.J. : Erlbaum.

Artigue, M. (1990). Ingénierie didactique. IN Recherches en didactique des mathématiques, Vol. 9 (3), pp. 281-308.

Artigue, M. (1994). Didactical engineering as a framework for the conception of teaching products. IN Didactics of Mathematics as a Scientific Discipline, Biehler et alii (Éds.), Kluwer Academic Publishers, pp. 27-39.

Bergeron, J., Herscovics, N. (1989). Un modèle de la compréhension pour décrire la construction de schèmes conceptuels mathématiques. IN Actes de la 41 ème rencontre de la Commission Internationale pour l'étude et l'amélioration de l'enseignement des mathématiques. Bruxelles, pp. 139-147.

Brousseau, G. (1998). Théorie des situations didactiques, Grenoble: La Pensée Sauvage, textes rassemblés par N. Balacheff, M. Cooper, R. Sutherland et V. Warfield.

Brousseau, G., Centeno, J. (1991). Rôle de la mémoire didactique de l'enseignant. IN Recherches en didactique des mathématiques, Vol. 11/2.3., pp. 167-210. 
Brown, S.I. (1986). La résolution de problèmes et la formation des enseignants : un compromis humaniste entre deux extrêmes. IN Etudes sur l'enseignement des mathématiques, La formation des professeurs de mathématiques de l'enseignement secondaire, R. Morris (éd.), Vol. 4, Paris : UNESCO, pp. 17-45.

Brown, J.S., Burton, R.R. (1978). Diagnostic models for procedural bugs in basic mathematical skills. IN Cognitive Science, Vol. 2, pp. 155-192.

Brun, J. (1994). Évolution des rapports entre la psychologie du développement cognitif et la didactique des mathématiques. IN Vingt ans de didactique des mathématiques en France - Hommage à Guy Brousseau et Gérard Vergnaud, M. Artigue, R. Gras, C. Laborde, P. Tavignot (Éds.), Grenoble : La Pensée Sauvage, pp. 67-83.

Centeno, J. (1995). La mémoire didactique de l'enseignant. Thèse Posthume. Bordeaux : LADIST.

Chevallard, Y. (1988). Notes sur la question de l'échec scolaire, Publications de l'IREM d'Aix Marseille, no. 13.

Chevallard, Y. (1991). La transposition didactique : du savoir savant au savoir enseigné. Grenoble : La Pensée Sauvage, $2^{\mathrm{e}}$ édition augmentée.

Chevallard, Y. (1996). Concepts fondamentaux de la didactique : perspectives apportées par une approche anthropologique. IN Didactique des mathématiques, J. Brun (Éd.), Lausanne : Delachaux et Niestlé, pp. 145-196.

Coppé, S. (1995). Types de connaissances mises en œuvre par l'élève dans la détermination de la composante publique de son travail. IN Différents types de savoirs et leur articulation, G. Arsac, J. Gréa, D. Grenier, et A. Tiberghien (Éds.), Grenoble : La Pensée Sauvage, pp. 129-144.

Conne, F. (1992). Savoir et connaissance dans la perspective de la transposition didactique. IN Recherches en didactique des mathématiques, Vol. 12/2.3, pp. 221-270.

Conne, F. (1996). Savoir et connaissance dans la perspective de la transposition didactique. IN Didactique des mathématiques, J. Brun (Éd.), Lausanne : Delachaux et Niestlé, pp. 275-338.

Conne, F. (1999). Faire des maths, faire faire des maths et regarder ce que ça donne. IN Le cognitif en didactique des mathématiques, G. Lemoyne, F. Conne (Éds.), Montréal : Les Presses de l’Université de Montréal, pp. 31-69.

Conne, F., Brun, J. (1991). Une analyse des brouillons de calcul d'élèves confrontés à des items de divisions écrites. IN Proceedings of the Fifteenth PME Conference, Vol. I, pp. 239-246.

DeBlois, L. (1996). Une analyse conceptuelle de la numération de position au primaire. IN Recherches en didactique des mathématiques, Vol. 16/1, pp. 71-127. 
Deloche, G., Seron, X. (1987). Numerical Transcoding : A General Production Model. IN Mathematical Disabilities, G. Deloche et X. Seron (Éds.), Hillsdale, N.J. : Erlbaum, pp. 137-170.

Deneau, C., Thivierge-Ayotte, L., Schmidt, S. (2001). Les significations accordées au signe « égal » et aux égalités arithmétiques par des élèves en difficulté grave d'apprentissage. IN Instantanés mathématiques, printemps, Vol. 4-12.

Farhnam-Diggory, S. (1979). Learning Disabilities, Guilford, London and Worcester : Billing \& Sons.

Faas, L.A. (1980). Children with learning problems - A handbook for Teachers, Boston : Houghton Mifflin.

Favre, J.-M. (1993). Élaborer une démarche d'enseignement par l'observation de la formation et de l'évolution d'un concept : la multiplication. IN Grand N, Vol. 53, pp. 27-37.

Favre, J.-M. (1997). L'échec, le temps, la multiplication. Mémoire de License, Université de Genève, Faculté de Psychologie et des sciences de l'éducation.

Favre, J.-M. (1999). La mathématique et le cognitif : deux chimères pour l'enseignant. IN Le cognitif en didactique des mathématiques, G. Lemoyne et F. Conne (Éds.), Montréal : Presses de l'Université de Montréal, pp. 235-261.

Franklin, W. (2000). Students at Promise and Resilient : A Historical Look at Risk Student. IN Schooling students placed at risk. Research, Policy, and Practice in the Education of Poor and Minority Adolescents, M.G. Sanders (Éd.), Hillsdale, N.J. : Erlbaum, pp. 3-16.

Gaudreau, A., Lemoyne, G., Poirier, L. (2001). Interactions enseignants-chercheurs dans la conception, la mise à l'essai et l'analyse de situations d'enseignement des mathématiques en classe spéciale. IN L'éducation au tournant du nouveau millénaire, G. Lemoyne et C. Lessard (Éds.), Université de Montréal, FSE, vice-décanat à la recherche, pp. 141-166.

Ginsburg, H. (1977). Children's Arithmetic : The Learning Process. New York : Van Nostrand.

Jaulin-Mannoni, F. (1965). La rééducation du raisonnement mathématique, Paris : Les Éditions Sociales Françaises.

Julo, J. (1995). Représentation de problèmes et réussite en mathématiques Un apport de la psychologie cognitive à l'enseignement, Rennes : Presses Universitaires de Rennes, Collection Psychologie.

Hinsley, D.A., Hayes, J.R., Simon, H.A. (1978). From words to equations. Meaning and representation in algebra word problems. IN Cognitive processes in comprehension, M. Just and P. Carpenter (Éds.), Hillsdale, NJ : Erlbaum, pp. 89-106.

Kintsch, W. (1977). Memory and Cognition, New York : Wiley. 
Krutetskii, V.A. (1976). The psychology of mathematical abilities in school children, Chicago : University of Chicago Press.

Lemoyne, G., Giroux, J., Biron, D. (1990). Connaissances utilisées par des élèves de 8 à 12 ans dans la formulation de problèmes arithmétiques concrets. IN European journal of psychology of education, Vol. 5(3), pp. 273-291.

Leong, C.K. (1982). Promising areas of research into learning disabilities with emphasis on reading disabilities. IN Theory and Research in Learning Disabilities, J.P. Das, R.F. Mulcahy and A.E. Wall (Éds.), New York : Plenum Press, pp. 3-26.

Matte, C. (1984). L'implantation d'un changement de politique à l'égard des enfants en difficulté d'apprentissage. Mémoire de maîtrise inédit, Université de Montréal.

Mayer, R.E. (1981). Frequency norms and structural analysis of algebraic story problems into families, categories, and templates. IN Instructional Science, Vol. 10, pp. 125-175.

Mayer, R.E. (1985). Mathematical ability. IN Human abilities : An information processing approach, R.J. Sternberg (Éd.), New York: Freeman, pp. 127-150.

Mercier, A. (1992). L'élève et les contraintes temporelles de l'enseignement, un cas en calcul algébrique. Thèse de Doctorat, Université de Bordeaux I, Bordeaux.

Mercier, A. (1995). Le traitement public d'éléments privés du rapport des élèves aux objets de savoir mathématiques. IN Différents types de savoirs et leur articulation, G. Arsac, J. Gréa, D. Grenier, A. Tiberghien (Éds.), Grenoble : La Pensée Sauvage, pp. 145-169.

Mercier, A. (1998). La participation des élèves à l'enseignement. IN Recherches en didactique des mathématiques, Vol. 18/3, pp. 279-310.

Mounoud, P. (1985). La notion de représentation en psychologie génétique. IN Psychologie française, Vol. 30, pp. 253-259.

Montague, M. (1992). The effects of cognitive and metacognitive strategy instruction on mathematical problem solving of middle school students with learning disabilities. IN Journal of Learning Disabilities, Vol. 23, pp. 230-248.

Montague, M. (1997). Effectiveness of Solve it. IN Journal of Learning Disabilities, Vol. 30, pp. 164-177.

Montague, M., Warger, C., Morgan, T.H. (2000). Solve it. Strategy Instruction to Improve Math. P.S. IN Learning Disabilities Research and Practice, Vol. 15(2), pp. 110-116.

Newell, A., Simon, H.A. (1972). Human Problem Solving, Englewoods-Cliffs, N.J. : Prentice-Hall. 
Perrin-Glorian, M.-J. (1993). Questions didactiques soulevées à partir de l'enseignement des mathématiques dans les classes faibles. IN Recherches en didactique des mathématiques, Vol. 13, no.1/2, Grenoble : La Pensée sauvage, pp. 5-118.

Pesenti, M., Seron, X. (2000). Neuropsychologie des troubles du calcul et du traitement des nombres, Marseille : Solal, Collection Neuropsychologie.

Polya, G. (1965). Comment poser et résoudre des problèmes, Paris : Dunod.

Richard, J.F. (1990). Les activités mentales : comprendre, raisonner, trouver des solutions, Paris : Armand Colin.

Riley, M.S., Greeno, J.G., Heller, J.I. (1983). Development of Children's Ability in Arithmetic. IN The Development of Mathematical Thinking, H.P. Ginsburg (Éd.), New York : Academic Press

Rosar, D., Van Nieuwenhoven, C., Jonnaert, P. (2001). Les fractions, comment mieux comprendre les difficultés rencontrées par les élèves? IN Instantanés mathématiques, hiver, pp. 4-16.

Rouchier, A. (1991). Étude de la conceptualisation dans le système didactique en mathématiques et informatique élémentaires : proportionnalité, structures itérativo-récursives, institutionnalisation. Thèse de doctorat d'Etat, Université d'Orléans.

Rouchier, A. (1999). La prise en compte du cognitif : jalons pour une évolution. IN Le cognitif en didactique des mathématiques, G. Lemoyne et F. Conne (Éds.), Montréal : Les Presses de l’Université de Montréal, pp. 177-195.

Rumelhart, D., Ortony, A. (1977). The representation of knowledge in memory. IN Schooling and the acquisition of knowledge, R. Anderson, R. Spire and W. Montague (Éds.), Hilsdale, NJ : Erlbaum.

Sarrazy, B. (1996). La sensibilité au contrat didatique - Rôle des Arrière-plans dans la résolution de problèmes darithmétique au cycle 3 . Thèse de doctorat inédite, Université Bordeaux I.

Schoenfeld, A.H. (1985). Mathemaatical Problem Solving, Orlando, FL : Academic Press.

Schoenfeld, A.H. (1987). Cognitive Science and Mathematics Education, Hillsdale NJ : Erlbaum.

Sensevy, G. (1998). Institutions didactiques - étude et autonomie de l'école élémentaire. Paris : Presses Universitaires de France.

Tardif, J., Presseau, A. (2000). L'échec scolaire en Amérique du Nord : un phénomène insidieux pour un grand nombre d'enfants et d'adolescents. IN Revue Française de Pédagogie, Vol. 130, pp. 89-105.

Terman, L., Merrill, M. (1960). Stanford-Bient Intelligence Scale. Boston : Houghton Mifflin Company, (édition révisée). 
Vergnaud, G. (1981). L'enfant, la mathématique et la réalité. Berne : Peter Lang.

Vergnaud, G. (1983). Multiplicative Structures. IN Acquisition of Mathematical Concepts and Processes, R. Lesh et M. Landau (Éds), New York : Academic Press, pp. 127-174.

Vergnaud, G. (1991). Langage et pensée dans l'apprentissage des mathématiques. IN Revue française de pédagogie, Vol. 96, pp. 79-86.

Vergnaud, G. (1996). Au fond de l'apprentissage, la conceptualisation. IN Actes de la VIII école et université d'été de didactique des mathématiques, R. Noirfalise, M.J. Perrin-Glorian (Éds.), IREM de Clermont-Ferrand, pp. 174-185.

Vygotsky, L.S. (1978). Mind in society. Cambridge, MA : Harvard University Press.

Wechsler, D. (1974). Wechsler Intelligence Scale for Children-Revised. New York : The Psychological Corporation.

Wong, B.Y.L. (1992). Contemporary Intervention Research in Learning Disabilities. An International Perspective, New York : Stringer-Verlag. 